\title{
MÉTODOS PARA A SUPERAÇÃO DA DORMÊNCIA FISIOLÓGICA DE Caryocar brasiliense Camb.
}

\author{
Jeferson Luiz Dallabona Dombroski ${ }^{1}$, Renato Paiva ${ }^{2}$, João Mauricio Cavalcante Alves ${ }^{3}$, Breno Regis Santos ${ }^{4}$, \\ Rairys Cravo Nogueira ${ }^{5}$, Patrícia Duarte de Oliveira Paiva ${ }^{6}$, Sandro Barbosa ${ }^{7}$
}

(received: August 24, 2009; accepted: February 26, 2010)

\begin{abstract}
RESUMO: O pequizeiro é uma frutífera nativa dos cerrados brasileiros com grande potencial econômico. Neste trabalho, objetivouse avaliar o efeito de substâncias potencialmente estimuladoras da germinação. As sementes foram extraídas dos caroços e colocadas para germinar em rolos de papel embebidos com as seguintes soluções: água destilada (Controle); $2 \mathrm{mmol} \mathrm{L}^{-1} \mathrm{KNO}_{3}(\mathrm{Nitrato}) ; 2 \mathrm{mmol} \mathrm{L}{ }^{-1}$ Etephon (ET); $1 \mathrm{mmol} \mathrm{L}^{-1} \mathrm{GA}_{3}(\mathrm{GA}) ; 1 \mathrm{mmol} \mathrm{L}^{-1} \mathrm{GA}_{3}+2 \mathrm{mmol} \mathrm{L}^{-1}$ Etephon $(\mathrm{GA}+\mathrm{ET})$. Os resultados de percentagem de germinação e o tempo médio para germinação foram, respectivamente, de 54,0\% e 9,3 dias no tratamento GA; 47,3\% e 11,0 dias no tratamento GA + ET; 32,0\% e 12,2 dias no tratamento Controle; 30,7\% e 13,1 dias no tratamento Etephon e 20,1\% e 13,1 dias no tratamento Nitrato. No tratamento GA + ET houve queda da taxa de germinação até os nove dias da semeadura, com relação ao tratamento GA, o que indica uma possível inibição da germinação de sementes de pequizeiros pela presença de etileno.
\end{abstract}

Palavras-chave: Caryocaraceae, quebra de dormência, tempo médio para germinação.

\section{PHYSIOLOGIC DORMANCY BREAKING METHODS OF Caryocar brasiliense Camb.}

\begin{abstract}
Caryocar brasiliense is a native species from Brazilian cerrado that has a considerable economic potential. The aim of this work was verifying the effect of substances with potential for seed germination stimulation. Seeds were extracted from the endocarps and set to germinate in paper rolls moistened with the following solutions: distilled water (Control); $2 \mathrm{mmol} \mathrm{L}^{-1} \mathrm{KNO}_{3}$

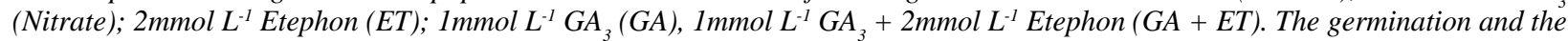
germination mean time were, respectively, $54.0 \%$ and 9.3 days in the GA treatment, $47.3 \%$ and 11.0 days in the GA + ET treatment, $32.0 \%$ and 12.2 days in the Control treatment, $30.7 \%$ and 13.1 days in the Etephon treatment, and $20.1 \%$ and 13.1 days in the Nitrate treatment. Low germination rates were observed in the GA +ET treatment up to the ninth day from sowing, when compared to the GA treatment, what indicates a possible seed germination inhibition due to ethylene presence.
\end{abstract}

Key words: Caryocaraceae, dormancy breaking, germination mean time.

\section{INTRODUÇÃO}

Uma espécie que representa o potencial econômico das plantas de cerrados é o pequizeiro (Caryocar brasiliense), por apresentar madeira de ótima qualidade usada na fabricação de móveis e ferramentas (BRASIL, 1985), frutos usados como medicamentos (ALMEIDA \& SILVA, 1994; OLIVEIRA et al., 1970) e, principalmente, na culinária, sendo considerado um suplemento alimentar importante para as populações de baixa renda (BRASIL, 1985; HERINGER, 1970; RIBEIRO et al., 1986; SENA, 1985, 1989).

O pequizeiro sofre pressões de extinção provocadas pelo extrativismo predatório dos frutos (ARAUUJ, 1995), afetando a propagação natural e pela diminuição das populações nativas (BRASIL, 1985), substituídas por monocultivos e pastagens. Além disso, as sementes apresentam forte dormência (ARAUJO, 1995; MIRANDA

\footnotetext{
${ }^{1}$ Engenheiro Agrônomo, Professor Dr. em Ciências Biológicas - Universidade Federal Rural do Semi Árido - BR 110 , km 47 - Bairro Presidente Costa e Silva - Biofábrica Presidente Costa e Silva - 59628-680 - Mossoró, RN - jeferson@ufersa.edu.br

${ }^{2}$ Engenheiro Agrônomo, Professor PhD em Agronomia - Setor de Fisiologia Vegetal - Departamento de Biologia/DBI - Universidade Federal de Lavras/UFLA - Cx. P. 3037 - 37200-000 - Lavras, MG - renpaiva@dbi.ufla.br

${ }^{3}$ Engenheiro Agrônomo, Dr. em Recursos Genéticos e Melhoramento de Plantas, Bolsista FAPEMIG - Departamento de Biologia/DBI Universidade Federal de Lavras/UFLA - Cx. P. 3037 - 37200-000 - Lavras, MG - jmca-bio@ hotmail.com

${ }^{4}$ Engenheiro Agrônomo, Professor Dr. Fisiologia Vegetal - Departamento de Ciências Biológicas e da Terra/DCBT - Universidade Federal de Alfenas/UNIFAL-MG - Rua Gabriel Monteiro da Silva, 700 - 37130-000 - Alfenas, MG - brenors@yahoo.com.br ${ }^{5}$ Bióloga, Professora Dra. em Fisiologia Vegetal - Universidade Federal do Pará/UFPA - Rua Coronel José Porfírio, 2515 - $68372-040$ Altamira, PA - rairys@yahoo.com.br

${ }^{6}$ Engenheira Agrônoma, Professora Dra. em Fitotecnia - Departamento de Agricultura/DAG - Universidade Federal de Lavras/UFLA Cx. P. 3037 - 37200-000 - Lavras, MG - patriciapaiva@dag.ufla.br

${ }^{7}$ Biólogo, Professor Dr. em Genética e Melhoramento de Plantas - Departamento de Ciências Biológicas e da Terra/DCBT - Universidade Federal de Alfenas/UNIFAL - Rua Gabriel Monteiro da Silva, 700 - 37130-000 - Alfenas, MG - sandro@unifal-mg.edu.br
} 
et al., 1988), e baixos índices de germinação (ARAUJO, 1995; CARVALHO et al., 1994; CARVALHO \& MARTINS, 2009; MELO \& GONÇALVES, 2001; MIRANDAet al., 1988).

Como tantas outras espécies nativas, a exigência de tratamentos específicos, bem como as baixas taxas e velocidades de germinação, dificultam o trabalho em viveiros, aumentando o custo de produção das mudas e, principalmente, dificultando o planejamento e a condução dos plantios definitivos (CAMARGOS et al., 2008; MARTINS et al., 2008; OLIVEIRA et al., 2009; VILELA et al., 2008).

Nesse contexto, conduziu-se este trabalho, com o objetivo de verificar o efeito de substâncias estimuladoras da germinação de sementes de pequizeiro.

\section{MATERIAL E MÉTODOS}

Frutos de uma árvore matriz, oriundos do município de Paraopeba, MG (latitude $19^{\circ} 16^{\prime}$, longitude $44^{\circ} 24^{\prime}$ ), foram usados como fonte de sementes. $\mathrm{O}$ mesocarpo foi removido com auxílio de equipamento desenvolvido por Dombroski (1997), constituído por três escovas rotativas de aço de $10 \mathrm{~cm}$ acopladas à extremidade de uma haste com $60 \mathrm{~cm}$ que, por sua vez, foi acoplada a um motor elétrico com 3/4CV e 1750rpm. O conjunto foi montado na vertical, com as escovas para baixo, mergulhadas em lata de 18 litros repleta com água, em que foram colocados cerca de 300 pequis. Em seguida, os endocarpos foram removidos com auxílio de esmerilador, por meio de corte transversal, evitando-se danos às sementes.

Após remoção do endocarpo, as sementes foram

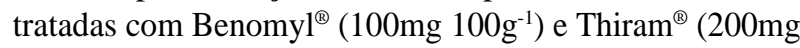
$\left.100 \mathrm{~g}^{-1}\right)$, e colocadas em câmara de germinação do tipo Mangelsdorf a $20^{\circ} \mathrm{C}$, em rolos de papel embebidos em: Água destilada (Controle); $2 \mathrm{mmol} \mathrm{L}^{-1} \mathrm{KNO}_{3}$ (Nitrato); $2 \mathrm{mmol} \mathrm{L}^{-1}$ Etephon - ethrel ${ }^{\circledR}(\mathrm{ET}) ; 1 \mathrm{mmol} \mathrm{L}^{-1} \mathrm{GA}_{3}(\mathrm{GA})$; e na combinação de $1 \mathrm{mmol} \mathrm{L}^{-1} \mathrm{GA}_{3}+2 \mathrm{mmol} \mathrm{L}^{-1}$ Etephon $(\mathrm{GA}+\mathrm{ET})$. Foram usadas cinco repetições por tratamento, com 30 sementes cada, em um delineamento inteiramente casualizado.

Aos 20 dias após o início do experimento, as sementes remanescentes em todos os tratamentos foram imersas por 24 horas em uma solução de $\mathrm{GA}_{3}$ a 2,0 $\mathrm{mmol} \mathrm{L}^{-1}$. Nessa etapa, em função do número reduzido de sementes nos tratamentos com giberelina e na combinação giberelina + Etephon $\left(\mathrm{GA}_{3}\right.$ e $\left.\mathrm{GA}_{3}+\mathrm{ET}\right)$, as sementes foram agrupadas em uma única repetição por tratamento otimizando o procedimento experimental.

A germinação foi avaliada a partir do terceiro dia após a instalação, a cada dois dias, até os 19 dias, quando as sementes foram agrupadas em uma única repetição por tratamento. A partir dessa data a avaliação foi feita a cada dois dias até os 32 dias, sendo feita uma avaliação final aos 42 dias, uma vez que testes preliminares mostraram que esse período era suficiente para as análises desejadas. Foram determinadas as percentagens médias de germinação aos 9 (G9) e aos 19 dias (G19), os tempos médios para germinação (TMG), segundo Edmond \& Drapala (1958), e as percentagens médias de sementes deterioradas (DET) e de sementes dormentes (DOR). Foram consideradas dormentes as sementes que se mantiveram firmes ao final do experimento, e consideradas deterioradas as sementes com perda de consistência e mau cheiro característico de bacteriose.

A normalidade dos resíduos foi avaliada pelo teste de Lilliefors e a homogeneidade das variâncias pelo teste de Cochran \& Barttlet a 5\%. As médias foram comparadas pelo Teste de Duncan a 10\%. Foi calculado o intervalo de confiança da média ao nível de $10 \%$. Os resultados de G9 foram transformados pela função G9* = $\ln (\mathrm{G} 9)$.

\section{RESULTADOS E DISCUSSÃO}

Os maiores valores de germinação acumulada, o menor número de sementes dormentes e o menor TMG foram observados nos tratamentos GA e GA+ET (Tabela 1). A germinação iniciou aos cinco dias, sendo observados picos de germinação entre 7 e 17 dias, os quais foram maiores nos tratamentos GA e GA + ET (Figura 1), e entre 26 e 28 dias para os tratamentos Etephon, Nitrato e Controle (Figura 1), ou seja, entre 8 a 10 dias após a segunda aplicação de $\mathrm{GA}_{3}$. Esses resultados indicam estímulo do $\mathrm{GA}_{3}$ sobre a germinação, semelhante ao que foi observado em trabalhos anteriores (DOMBROSKI, 1997; OLIVEIRA, 2002), e sugere que o tempo necessário entre 7 e 11 dias para resposta ao $\mathrm{GA}_{3}$ para sementes de pequizeiros.

Na Tabela 1, pode-se observar que, até nove dias após a instalação do experimento, a germinação das sementes mantidas na presença de Nitrato, Etephon e da combinação entre $\mathrm{GA}_{3}$ + ET mostrou-se estatisticamente similar ao controle (água destilada) divergindo do efeito da aplicação de $\mathrm{GA}_{3}$ durante esse período. Isso refletiu no aumento do TMG, que foi semelhante ao alcançado no tratamento com água destilada. A partir dos nove dias a inibição desapareceu, e as sementes retomaram a germinação alcançando valores próximos ao do tratamento GA (Tabela 1). É possível que a inibição provocada pelo etephon tenha sido eliminada após a dispersão do etileno. O etephon é uma substância química que ao ser metabolizado pelos tecidos de plantas libera etileno nos níveis citoplasmáticos (GEORGE, 2008). Contudo, o etileno expressa sua ação de forma temporária por sua característica volátil.

Cerne, Lavras, v. 16, n. 2, p. 131-135, abr./jun. 2010 
Tabela 1 - Aplicação de nitrato, etephon (ET) e GA 3 (GA) na percentagem de sementes de pequizeiro germinadas aos nove (G9) e aos 19 (G19) dias, tempo médio para germinação (TMG), e percentagens de sementes deterioradas (DET) e dormentes (DOR) aos 19 dias após a semeadura.

Table 1 -Nitrate, etephon (ET) and $\mathrm{GA}_{3}(G A)$ application on the percentage of Caryocar brasiliense seeds germinated at nine (G9) and 19 (G19) days, average time for germination (TMG), and deteriorated seed percentages (DET) and dormant (DOR) at 19 days after sowing.

\begin{tabular}{lrrrrrrrrrrr}
\hline Tratamento & \multicolumn{2}{c}{ G9* $(\%)$} & \multicolumn{2}{c}{ G19 $(\%)$} & \multicolumn{2}{c}{ TMG $($ dias $)$} & \multicolumn{2}{c}{ DET $(\%)$} & & DOR $(\%)$ \\
\hline Água destilada & $7,3 \pm 4,3$ & bc & $32,0 \pm 7,2$ & b & $12,2 \pm 0,9$ & c & $44,7 \pm 8,6$ & a & & $23,3 \pm 7,3$ & a \\
Nitrato & $4,7 \pm 3,2$ & c & $20,1 \pm 7,1$ & cc & $13,1 \pm 0,8$ & c & $50,0 \pm 10,3$ & a & $29,9 \pm 8,6$ & a \\
Etephon & $3,3 \pm 2,7$ & c & $30,7 \pm 8,8$ & bc & $13,1 \pm 1,1$ & c & $48,0 \pm 8,9$ & a & $21,3 \pm 5,7$ & a \\
$\mathrm{GA}_{3}$ & $32,7 \pm 6,4$ & a & $54,0 \pm 7,5$ & a & $9,3 \pm 0,7$ & a & $38,0 \pm 6,8$ & a & $8,0 \pm 3,0$ & b \\
$\mathrm{GA}_{3}+$ ET & $8,0 \pm 4,8$ & b & $47,3 \pm 7,8$ & a & $11,0 \pm 0,3$ & b & $46,7 \pm 7,5$ & a & & $6,0 \pm 2,2$ & b \\
\hline
\end{tabular}

Médias seguidas pela mesma letra não diferem estatisticamente entre si, pelo teste de Duncan, a $10 \%$.
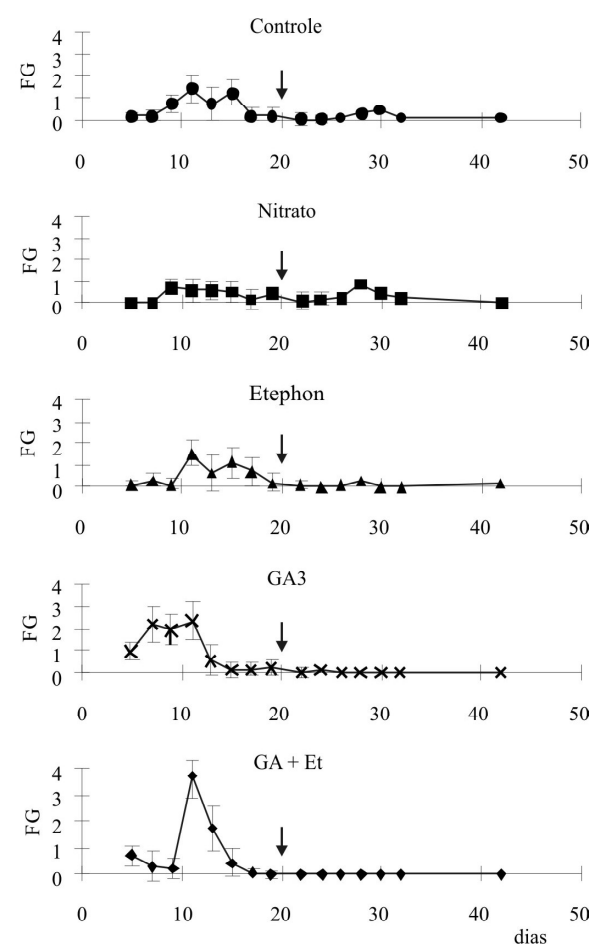

Figura 1 - Frequência absoluta de germinação (FG) de sementes de pequizeiro após tratamentos com água, nitrato, etephon e $\mathrm{GA}_{3}$. A seta indica o momento da aplicação de $2 \mathrm{mmol} \mathrm{L}^{-1} \mathrm{GA}_{3}$, aos 20 dias. As barras de erro representam o erro padrão das médias a $10 \%$.

Figure 1 - Absolute germination frequency $(G F)$ of Caryocar brasiliense seeds after treatment with water, nitrate, etephon and $\mathrm{GA}_{3}$. The dart indicates the moment of application of $2 \mathrm{mmol}$ $L^{-1} G A_{3}$ at 20 days. The bars represent the error average pattern at 10
O etileno pode afetar a germinação e a dormência de sementes de várias espécies, podendo ser estimulador, como para Striga asiatica e Orobanche racemosa; inibidor, como para Plantago major e Chenopodium rubrum, ou não efetivo (CORBINEAU \& CÔME, 1995). Os resultados obtidos nesse experimento indicam que a dose usada de etephon inibiu o estímulo provocado pela aplicação de ácido giberélico sobre a germinação do pequizeiro durante nove dias.

A aplicação de $2 \mathrm{mmol} \mathrm{L}^{-1}$ de nitrato de potássio mostrou efeitos similares àqueles observados tanto no controle quanto no tratamento com etephon nas taxas de germinação (Tabela 1, Figura 1) diferindo de $\mathrm{GA}_{3}$ e da interação $\mathrm{GA}_{3}$ + ET, que apresentaram redução da TGM.

O nitrato é nutriente importante para a germinação de algumas espécies, como Sisymbrium officinale (HILHORST, 1996), sendo conhecido pelo estímulo à germinação, e usado rotineiramente para quebra de dormência de sementes em muitas espécies comerciais (BRADBEER, 1988). Os mecanismos da sua ação são desconhecidos (EGLEY \& DUKE, 1987) e, segundo Fenner (1995), é possível que a resposta ao nitrato seja um mecanismo de detecção das estações do ano, pois ocorre aumento da disponibilidade de nitrato nas épocas chuvosas e com temperaturas altas. Porém, não foi encontrada na literatura nenhuma referência que mencione inibição da germinação pelo nitrato.

$\mathrm{O}$ resultado do efeito da aplicação de $\mathrm{GA}_{3}$, nas sementes remanescentes dos tratamentos, encontra-se na Tabela 2. A ocorrência de sementes viáveis é um indício de que a concentração usada de $\mathrm{GA}_{3}$ foi insuficiente para a quebra de dormência das sementes remanescentes, ou que existem outros mecanismos de dormência embrionária pouco afetados pela adição de $\mathrm{GA}_{3}$

Cerne, Lavras, v. 16, n. 2, p. 131-135, abr./jun. 2010 
Tabela 2 - Efeito da aplicação de $\mathrm{GA}_{3}$ (aos 20 dias) sobre o número inicial de sementes de pequizeiro germinadas (GER) e dormentes (DOR) aos 42 dias após tratamento inicial com nitrato, etephon, $\mathrm{GA}_{3}$ ou $\mathrm{GA}_{3}+$ etephon.

Table 2 - Effect of $G A_{3}$ application (at 20 days) on the initial number of germinated (GER) seeds of Caryocar brasiliense and dormant $(D O R)$ at 42 days after initial treatment with nitrate, etephon, $G_{3}$ or $G_{3}+$ etephon.

\begin{tabular}{lccc}
\hline Tratamento & Número de sementes & GER & DOR \\
\hline Água destilada & 35 & 10 & 25 \\
Nitrato & 45 & 22 & 23 \\
Etephon & 32 & 7 & 25 \\
$\mathrm{GA}_{3}$ & 8 & 1 & 7 \\
$\mathrm{GA}_{3}+\mathrm{Et}$ & 1 & 0 & 1 \\
\hline
\end{tabular}

\section{CONCLUSÕES}

A aplicação de $\mathrm{GA}_{3}$ provocou diminuição do tempo médio para germinação e aumento da taxa de sementes germinadas.

A aplicação de nitrato de potássio inibiu a germinação de sementes de pequizeiro.

O etephon, quando aplicado simultaneamente com GA3, provocou inibição, por alguns dias, no efeito estimulador do $\mathrm{GA}_{3}$.

\section{AGRADECIMENTOS} FAPEMIG.

Os autores agradecem o apoio do CNPq, CAPES e

\section{REFERÊNCIAS BIBLIOGRÁFICAS}

ALMEIDA, S. P.; SILVA, J. A. Piqui e buriti: importância alimentar para a população dos cerrados. Planaltina: EmbrapaCPAC, 1994. 38 p. (Documentos, 54).

ARAUJO, F. D. A review of Caryocar brasiliense Camb. (Caryocaraceae): an economically valuable species of the central Brazilian cerrados. Economic Botany, New York, v. 49, n. 1, p. 40-48, 1995.

BRADBEER, J. W. Seed dormancy and germination. Glasgow: Blackie, 1988. 146 p.

BRASIL. Ministério da Indústria e do Comércio. Secretaria de Tecnologia Industrial. Produção de combustíveis líquidos a partir de óleos vegetais. Brasília, 1985. 194 p.

CAMARGOS, V. N.; CARVALHO, M. L. M. de; ARAÚJO, D. V. de; MAGALHÃES, F. H. L. Superação da dormência e avaliação da qualidade fisiológica de sementes de Sesbania virgata.
Ciência e Agrotecnologia, Lavras, v. 32, n. 6, p. 1858-1865, nov./dez. 2008.

CARVALHO, C. G. S. e; CÔRTES, R. A.; CARNEIRO, I. F.; BORGES, J. D. Efeito de diferentes tratamentos na germinação do pequi (Caryocar brasiliense Camb.). Acta Botânica Brasileira, São Paulo, v. 8, n. 1, p. 109-120, 1994.

CARVALHO, D. A. de; MARTINS, F. R. Shrub and tree species composition in the cerrados of south west Minas Gerais. Cerne, Lavras, v. 15, n. 2, p. 142-154, abr./jun. 2009.

CORBINEAU, F.; CÔME, D. Control of seed germination and dormancy by the gaseous environment. In: KIGEL, J.; GALILI, G. Seed development and germination. New York: M. Dekker, 1995. 853 p.

DOMBROSKI, J. L. D. Estudos sobre a propagação do pequizeiro (Caryocar brasiliense Camb.). 1997. 78 f. Dissertação (Mestrado em Agronomia) - Universidade Federal de Lavras, Lavras, 1997.

EDMOND, J. D.; DRAPALA, W. J. Effects of temperature, sand and soil, and acetone on germination of Okra seeds. Proceedings of the American Society of Horticultural Science, New York, v. 71, p. 428-434, June 1958.

EGLEY, G. H.; DUKE, S. O. Physiolgy of weed seed dormancy and germination. In: DUKE, S. O. Weed physiology: reproduction and ecophysiology. Boca Raton: CRC, 1987. v. 1, p. 27-64.

FENNER, M. Ecology of seed banks. In: KIGEL, J.; GALILI, G. Seed development and germination. New York: M. Dekker, 1995. p. 507-528.

Cerne, Lavras, v. 16, n. 2, p. 131-135, abr./jun. 2010 
GEORGE, E. F.; HALL, M. A.; KLERK, G. Plant propagation by tissue culture: the background. London: Exegetics, 2008. v. $1,501 \mathrm{p}$.

HERINGER, E. P. Pequizeiro (Caryocar brasiliense Cambess). Brasil Florestal, Rio de Janeiro, v. 1, p. 28-31, fev. 1970.

HILHORST, H. W. M. An integrating model for seed dormancy. In: LANG, G. A. Plant dormancy: physiology, biochemistry and molecular biology. London: CAB International, 1996. chap. 24 , p. $341-360$.

MARTINS, C. C.; MACHADO, C. G.; CAVASINI, R. Temperatura e substrato para o teste de germinação de sementes de pinhão-manso. Ciência e Agrotecnologia, Lavras, v. 32, n. 3, p. 863-868, maio/jun. 2008.

MELO, J. T. de; GONÇALVES, A. N. Inibidores de germinação em frutos e sementes de pequi. Boletim de Pesquisa e Desenvolvimento - Embrapa Cerrados, Planaltina, n. 23, p. 1-11, dez. 2001.

MIRANDA, J. de S.; SILVA, H.; MATOS, M. A. de O. Emergência e vigor de sementes de pequi submetidas a prétratamentos mecânicos e térmicos. In: CONGRESSO BRASILEIRO DE FRUTICULTURA, 9., 1987, Campinas. Anais... Campinas: Sociedade Brasileira de Fruticultura, 1988. p. $647-651$.

OLIVEIRA, H. M.; NERY, F. C.; ALVARENGA, A. A. de; BARBOSA, J. P. D.; CARVALHO, D. D. C. Comportamento germinativo de sementes de Talisia subalbens (Mart.) Radlk.
(Sapindaceae) submetidas a diferentes temperaturas e condições de secagem. Ciência e Agrotecnologia, Lavras, v. 33, n. 2, p. 391-396, mar./abr. 2009.

OLIVEIRA, M. M. de; SAMPAIO, R. P.; GIORGI, W.; GILBERT, B.; MORS, W. Caryocar brasiliense: isolamento e identificação de algumas substâncias: atividade biológica sobre o sarcoma 180. Arquivos do Instituto Biológico, São Paulo, v. 37, n. 1, p. 25-27, jan. 1970 .

OLIVEIRA, S. S. Efeito de giberelina, fungicida, tratamentos mecânicos e período de armazenamento sobre a germinação de sementes de pequizeiro. 2002.58 p. Dissertação (Mestrado em Agricultura Tropical) - Universidade Federal de Mato Grosso, Cuiabá, 2002.

RIBEIRO, J. F.; PROENÇA, C. E. B.; ALMEIDA, S. P. Potencial frutífero de algumas espécies nativas do cerrado. In: CONGRESSO BRASILEIRO DE FRUTICULTURA, 8., 1986, Brasília. Anais... Brasília: Embrapa-DDT/CNPq, 1986. v. 2, p. 491-500.

SENA, A. O grito do pequizeiro. O Estado de Minas, Belo Horizonte, 30 nov. 1985. Caderno Agropecuário, p. 5.

SENA, A. Pequi: a força da terra contra a devastação florestal. O Estado de Minas, Belo Horizonte, 4 fev. 1989. Caderno Agropecuário, p. 6-7.

VILELA, G. F.; CARVALHO, D. de; VIEIRA, F. de A. Fenologia de Caryocar brasiliense Camb. (Caryocaraceae) no alto Rio Grande, sul de Minas Gerais. Cerne, Lavras, v. 14, n. 4, p. $317-$ 329, out./dez. 2008. 